\title{
Response measures influence comparative effectiveness of biological drugs in rheumatoid arthritis
}

\author{
Helena Canhao ${ }^{*}$, Vasco Romao, Fernando Martins, Joao Eurico Fonseca \\ From 7th European Workshop on Immune-Mediated Inflammatory Diseases \\ Noordwijk aan Zee, the Netherlands. 28-30 November 2012
}

\section{Background}

Rheumatoid arthritis (RA) is a chronic, disabling inflammatory disease. Tumor necrosis factor (TNF) and interleukin-6 (IL-6) are key elements in the disease pathway and constitute the target of the most commonly used biological drugs. Infliximab, adalimumab, etanercept and golimumab are TNF inhibitors and tocilizumab is an IL-6-receptor antagonist.

The aim of this work was to assess comparative effectiveness of biological drugs for RA patients registered in Reuma.pt, using different validate tools.

\section{Methods}

We included RA first biological therapy users. Our primary outcome was EULAR good response at 6 months. Secondary outcomes were the proportion of patients in remission applying DAS28, CDAI and SDAI. Groups were compared using a multivariate logistic model.

\section{Results}

520 RA patients. 123 treated with adalimumab, 204 etanercept, 31 golimumab, 110 infliximab and 52 with tocilizumab. Mean age at biologics start was $54.4 \pm 12.5$ years and disease duration of $11.2 \pm 9.6$ years. $86.9 \%$ were females and $83.0 \%$ were either rheumatoid factor or ACPA seropositive. Baseline DAS was $5.3 \pm 1.2$ for adalimumab, $5.6 \pm 1.2$ for etanercept, $5.4 \pm 1.3$ for golimumab, $5.6 \pm 1.3$ for infliximab and $5.7 \pm 1.2$ for tocilizumab groups ( $\mathrm{p}=0.12$ ANOVA). At 6 months, DAS was $3.7 \pm$ 1.3 for adalimumab, $3.8 \pm 1.3$ for etanercept, $3.5 \pm 1.3$ for golimumab, $3.7 \pm 1.4$ for infliximab and $1.6 \pm 0.99$ for tocilizumab groups ( $<0.0001$ ANOVA). The probability of achieving EULAR good response at 6 months was modeled. Significantly higher rate of good response at 6 months was observed in tocilizumab compared to other groups with the exception of golimumab. DAS28 remission threshold was attained by a significantly higher number of patients treated with tocilizumab. Remission rates assessed by CDAI and SDAI did not show significant differences between groups.

\section{Conclusions}

Tocilizumab demonstrated the best efficacy, but the difference varied with the disease activity tool chosen. These results reflect the importance of the tool used in the assessment of treatment response and may be explained by the pronounced effect of tocilizumab on the reduction of erythrocyte sedimentation rate, considered only by DAS28 and not by the other scores.

Published: 28 November 2012

doi:10.1186/1479-5876-10-S3-P52

Cite this article as: Canhao et al:: Response measures influence comparative effectiveness of biological drugs in rheumatoid arthritis. Journal of Translational Medicine 2012 10(Suppl 3):P52. 